\title{
Clinical and ultrasonographic features of medullary thyroid microcarcinomas compared with papillary thyroid microcarcinomas: a retrospective analysis
}

\section{Xiaoyu Li}

Ultrasound Department, Rui Jin Hospital, Shanghai Jiao Tong University School of Medicine

\section{Wei Zhou}

Shanghai Jiao Tong University Medical School Affiliated Ruijin Hospital Department of Ultrasound

Weiwei Zhan ( $\sim$ shanghairuijinus@163.com )

Shanghai Jiao Tong University Medical School Affiliated Ruijin Hospital

\section{Research article}

Keywords: medullary thyroid carcinoma, papillary thyroid carcinoma, ultrasound

Posted Date: April 6th, 2020

DOI: https://doi.org/10.21203/rs.2.16733/v3

License: (c) (i) This work is licensed under a Creative Commons Attribution 4.0 International License.

Read Full License

Version of Record: A version of this preprint was published at BMC Medical Imaging on May 14th, 2020. See the published version at https://doi.org/10.1186/s12880-020-00444-9. 


\section{Abstract}

Background: To identify the sonographic features that help to differentiate medullary thyroid microcarcinomas (MTMCs) and papillary thyroid microcarcinomas (PTMCs). Methods: A total of 46 MTMCs in 41 patients and 136 PTMCs in 104 patients that were proved by surgery and pathology, were included in the study. The age and nodule size were analyzed by independent sample t test, and the gender, multiplicity and cervical lymph node metastases were analyzed by $\chi 2$ or Fisher's exact tests. Univariate analysis and multivariate logistic regression analysis were performed on the sonographic features of thyroid nodules, including location, shape, boundary, margin, peripheral halo ring, echogenicity, composition, calcifications and vascularization. Results: Compared with PTMCs, there were more MTMCs patients with cervical lymph node metastases $(P=0.040)$. There were no significant differences in age, gender, nodule size, multiplicity, location, boundary, margin, peripheral halo ring, echogenicity and microcalcification between MTMCs and PTMCs ( $>0.05$ for all). However, significant differences were found in shape $(P=0.000)$, composition $(P=0.032)$, macrocalcification $(P=0.004)$ and vascularity $(P=0.000)$ between the two groups. Conclusions: There was some overlapping of sonographic features of MTMCs and PTMCs. However, MTMCs tended to be $>50 \%$ solid composition, ovoid to round nodules with macrocalcifications and hypervascularity. Cervical lymph node metastases were more common in MTMCs patients.

\section{Background}

Due to the wide use of ultrasound (US), the detection rate of small thyroid nodules has increased, especially the nodules less than $10 \mathrm{~mm}[1,2]$. At present, many controversies still remain regarding the treatment of these small thyroid nodules with malignant features. Papillary thyroid microcarcinomas (PTMCs) are defined as papillary thyroid carcinomas (PTCs) less than $10 \mathrm{~mm}$ in diameter with a good prognosis. The American Thyroid Association (ATA) guidelines suggested that an active surveillance could be considered in some PTMC patients. It was not applied to medullary thyroid microcarcinomas (MTMCs), although the size is also smaller than $10 \mathrm{~mm}$. Medullary thyroid carcinomas (MTCs) derived from thyroid follicular $\mathrm{C}$ cells, which account for $3-4 \%$ of all thyroid malignancies, have a high recurrence rate and a poor prognosis [3]. MTCs are not sensitive to radiotherapy and chemotherapy. The 10-year mortality rates of MTCs reaches 13.5-38\% [4-6]. The early diagnosis and treatment of MTCs are critical to improve the disease-free survival and reduce the mortality rate. Therefore, it is very important to differentiate PTMCs from MTMCs.

Previous studies on the ultrasonographic features of small thyroid nodules mostly focused on PTMC, which usually presented as a solid and hyperechoic nodule with a taller-than-wide shape, an irregular margin and microcalcifications. However, due to the diversified sonographic manifestations and low incidence, the sonographic features of MTMCs have been reported in few studies with limited number of cases. To the best of our knowledge, there was only one report about the sonographic appearances of seven MTMCs that were compared with PTMCs [7]. To expand on these findings, this study was aimed to evaluate the ultrasonographic characteristics of the MTMCs, which may be useful to identify the 
sonographic features of MTMCs and provide useful information in the differential diagnosis of MTMCs and PTMCs.

\section{Methods}

\section{Patients}

This retrospective study was approved by the Institutional Review Board, with waiver of informed consent. A systematic review consisted of the MTMCs diagnosed from January 2008 to April 2017. A total of 41 patients with 46 MTMCs, which were surgically confirmed, were included in this study. Of the 41 patients, 35 patients $(85.37 \%)$ had a sporadic type of MTCs, 5 patients $(12.20 \%)$ presented with multiple endocrine neoplasia (MEN) types II A, and one patient (2.43\%) presented with MEN II B. As a control group, 104 patients with 136 PTMCs confirmed by post-operative pathology from January to June 2015 were included in this study.

\section{Sonography Imaging analysis}

All the patients were evaluated by US. US examinations were performed with Philips IU22 (New York), GE LOGIQ E9 (New York), or MyLab 90 (Italy), equipped with a 5-12 MHz linear array transducer. All the examinations were performed by two radiologists with more than 10 years of experience in thyroid imaging.

All the thyroid nodules were evaluated in the transverse and longitudinal plane by gray scale and color Doppler ultrasonography. The sonographic features of nodules were carefully evaluated, including: location, shape, boundary, margin, peripheral halo ring, echogenicity, composition, calcification, and vascularization. The cervical lymph nodes were also evaluated. All the US images were retrospectively analyzed by the same radiologist who was blinded to pathological results.

The location was determined as upper and lower pole. Shape was assessed as ovoid to round, taller than wide and irregular. Boundary was classified as clear or unclear. Margin was divided into smooth, spiculated and poorly defined. Peripheral halo ring was defined as absent or present. Compared with the adjacent thyroid tissue, the echogenicity was categorized into markedly hypoechoic, hypoechoic, and hyperechoic or isoechoic. According to the proportion of solid components, the composition was classified as solid and $>50 \%$ solid. Calcifications were recorded as absent or present. When calcification was observed, it was categorized into microcalcification (with diameter $<2 \mathrm{~mm}$ ) and macrocalcification (with diameter $\geq 2 \mathrm{~mm}$ or coexisted with microcalcifications). The nodule was considered as hypervascularity when it was similar to or more than the surrounding thyroid tissue [8]. The remaining nodules were regarded as avascularity.

\section{Data and Statistical Analyses}

Statistical analysis was performed by SPSS 20.0 software package (SPSS, Chicago, IL). And the statistical significance was determined as $\mathrm{P}<0.05$. 
The independent-sample t test was used to analyze age and size. Categories variables, including gender, lymph node metastases, location, composition, shape, margin, boundary, echogenicity, calcification, and vascularization, were analyzed by $\chi^{2}$ test or Fisher's exact test. The variables with statistical significance were included in the multivariate regression model. $B$, Wals $\chi^{2}$, ratio (OR) and $95 \% \mathrm{Cl}$ were recorded.

\section{Results}

\section{Clinical characteristics of MTMCs and PTMCs}

The clinical characteristics of patients were summarized in Table 1. There were no significant differences in age (41.78 \pm 16.39 years vs $44.07 \pm 10.43$ years, $P=0.323)$, sex (female: male ratio, $23: 18$ vs $70: 34$; $\mathrm{P}=0.205)$ and tumor size $(6.30 \pm 2.27 \mathrm{~mm}$ vs $6.06 \pm 2.16 \mathrm{~mm}, \mathrm{P}=0.605)$ between MTMCs and PTMCs group. The incidences of single nodule and lymph node metastases in the MTMCs group $(87.80 \%$ and $36.59 \%)$ were higher than those in PTMCs group (70.19\% and $20.19 \%)(P<0.05$ for both).

\section{Univariate analysis of US features}

The results of univariate analysis for US characteristics were summarized in Table 2. There were no significant differences in location, margin, peripheral halo ring and echogenicity between MTMCs and PTMCs groups $(P>0.05$ for all), however, a significant difference was found in shape, boundary, composition, calcification and vascularity between the two groups $(P<0.05$ for all).

\section{Multivariate Logistic regression analysis of US features}

The results of multivariate logistic regression analysis for US characteristics were summarized in Table 3. The proportion of MTMCs with ovoid to round shape (Fig. 1, 2) was higher than that of PTMCs (Fig.5) (54.35\% vs $9.56 \%, P=0.007$ ), but PTMCs with taller than wide shape (Fig. 4,6 ) were much more than MTMCs (Fig. 3) (59.56\% vs $19.57 \%, P=0.000)$. An unclear boundary seemed to be more commonly detected in PTMCs (Fig. 5) than in MTMCs, however, there was on significant difference $(86.03 \% \mathrm{vs}$ $43.48 \%, P=0.188$ ). There were more nodules with composition $>50 \%$ solid in MTMCs group compared with PTMCs (13.04\% vs 1.47\%, P=0.032). Calcifications were not commonly observed in both groups, when occurred, macrocalcifications were more often seen in MTMCs (Fig. 2) than in PTMCs (Fig. 5) (21.74\% vs 6.62\%, P=0.004). Microcalcifications were more frequently detected in PTMCs (Fig. 4), although there was no statistical significance $(10.87 \%$ vs $29.41 \%, P=0.214)$. Hypervascularity was observed in $58.70 \%$ of MTMCs, but only in $19.85 \%$ of PTMCs $(P=0.000)$.

\section{Discussions}

Previous studies had showed that MTCs patients were more common in females, with single nodule in the majority of cases $[9,10]$. In this study, there were no significant differences in age and tumor size between MTMCs and PTMCs. There was a slightly higher number of women than men in MTMCs patients in this study, however, there was also no statistical significance. In accord with previous reports 
$[9,10]$, most patients with MTMCs had a single lesion, and only 5 cases had two MTMCs nodules in the present study. PTCs often arise as multiple tumor foci, which are considered as the result of multiple synchronous primary tumors arising from independent clones. The incidence of multifocal PTMCs has been reported as 23.5\%-36.1\% [11-13]. In our study, multiple PTMCs were detected in $29.81 \%(31 / 104)$ cases, which was higher than that in MTMCs group. Our results showed that there was significantly different in lymph node metastases between MTMCs group and PTMCs group. In PTMCs group, 21 $(20.19 \%)$ patients had lymph node metastases, the incidence of which was similar to those in previous reports [14-16]. MTMCs were more likely to have lymph node metastases, which indicated the strong invasive characteristics of MTCs. This also suggests that although the incidence of MTCs is very low, early diagnosis is of great significance.

Zhou et al [7] found that MTMCs had no significant difference from PTMCs in internal composition, calcifications, echogenicity, margin, and shape $(P>0.05)$. In our study, there were no significant differences in location, margin, boundary, echogenicity, peripheral halo ring, and the presence of calcifications between the two groups $(P>0.05)$. But there were statistic differences in the characteristics as follows: internal composition, shape, calcification types and vascularity, which were not exactly consistent with the results in Zhou's. The possible reasons may be the different sample size, imaging instrument and evaluation standard of the ultrasonic characteristics.

Cystic change was not common in PTMCs and MTMCs [ 7, 10]. Our results also showed that the majority of cases in both MTMCs (86.96\%) and PTMCs (98.53\%) exhibited solid composition, however, $>50 \%$ solid composition was significantly more frequent in MTMCs than in PTMCs. In 46 MTMCs, $>50 \%$ solid composition was observed in $6(13.04 \%)$ lesions. Although the mechanism of cystic change is unclear, solid-cystic nodules might result from degeneration of solid nodules, with the accumulation of fluid probably due to intranodular necrosis. MTCs tended to display less differentiated and grow faster than PTCs, and cystic change in MTCs was most likely induced by necrosis as a result of cell proliferation that exceeds the available blood supply. Lee et al [10] also reported that cystic change was significantly more common in MTCs compared with in PTCs, and the tumor size had no significant relationship with cystic changes.

PTC usually had a large intensive fibrosis, and its compressibility was reduced, resulting in its standinglike morphology [17]. A taller-than-wide shape was regarded as an ultrasongraphic feature of PTMCs [18, 19]. In our study, it was also a common sign in PTMCs group, and the proportion was as high as $59.56 \%$. However, there was only 9 (19.57\%) cases with a taller-than-wide shape in MTMCs group, which indicated that it did not apply to MTMCs. Previous studies have shown that, regardless of size, the shape of MTCs was mostly round or ovoid rather than taller than wide $[20,21]$. Similar results were also observed in our series. The MTMCs nodules were more likely to show ovoid to round comparing with PTMCs, and the difference was statistically significant $(P=0.000)$.

Calcifications, especially microcalcifications, were regarded as a specific sign associated with thyroid malignancy. Kim et al [22] found that calcifications were not significantly different between PTCs and 
MTCs. The results of our study showed that calcifications were not common in both MTMCs group and PTMCs group, and the incidence of calcification was similar $(P=0.674)$. However, the type of calcification was different between the two groups. We found that microcalcifications were frequently seen in PTMCs, and macrocalcifications appeared more commonly in MTMCs. This might be related to the different mechanisms of calcification formation in MTCs and PTCs. Calcifications in MTCs are mainly due to the deposition of local calcium salts surrounded by amyloid substances, leading to the formation of coarse calcifications. However, calcifications in PTCs are mainly caused by psammoma bodies with the smaller diameter of 10 100 $\mu \mathrm{m}$, which are commonly manifested in round or concentric under the light microscope.

To the best of our knowledge, there were very limited literatures reported the blood supply of MTCs. Trimboli et al [23] reported that the percentage of intranodular vascularization in MTCs (25\%) was higher than that in PTCs (15\%), but with no significant difference. However, in the present study, MTMCs were more likely to show hypervascularity than PTMCs $(P=0.000)$. PTMCs were predominantly characterized by reduced blood supply, which might be due to the incompletely developed neoangiogenetic vascular bed in comparison with the uncontrolled cell proliferation typical of carcinoma [24]. In the present series, $80.15 \%$ of PTMCs were deficient in vascularity. According to the study reported by Lai et al [25], $72.4 \%$ of MTCs showed hypervascularity, which might be induced by the rapid division and growth of MTCs cells, resulting in a faster rate of proliferation than PTCs. In this study, abundant blood supply was also commonly detected in MTMCs (58.70\%), but the proportion was not as high as reported in the literature. This may be due to the relatively small number of blood vessels caused by the small size of the tumor.

There were some limitations in our study. First, this was a retrospective study with statistical bias. Second, because of the low incidence of MTCs, it took a long time to collect MTMCs cases. During this time, there has been a significant development in US technology, which is likely to have an impact on the evaluation of sonographic features. In addition, the sample size was small in MTMCs group, although there were more cases than those in the previous studies. A further study with a larger sample size should be performed to identify the sonographic features of MTMCs.

\section{Conclusions}

There was some overlapping of sonographic features of MTMCs and PTMCs, and it was difficult to accurately distinguish these two types of thyroid carcinomas. However, MTMCs were more likely to be ovoid to round nodules with $>50 \%$ solid composition, macrocalcifications and hypervascularity compared with PTMCs, and cervical lymph node metastases were more common in MTMCs patients.

\section{Abbreviations}

MTC: medullary thyroid carcinoma

MTMC: medullary thyroid microcarcinoma 
PTMC: papillary thyroid microcarcinoma

PTC: papillary thyroid carcinoma

US: ultrasound

MEN: multiple endocrine neoplasia

\section{Declaration}

\section{Ethics approval and consent to participate}

This retrospective study was approved by the Ethics Committee of the Ruijin Hospital, Shanghai Jiao Tong University School of Medicine. The procedures of reviewing the research were in line with the ethical standards of the institutional and national research committees.

\section{Consent for publication}

Not applicable.

\section{Availability of data and material}

All data in this study are available from the corresponding authors upon reasonable request.

\section{Competing interests}

The authors declare that they have no competing interests.

\section{Funding}

None.

\section{Authors' contributions}

$\mathrm{XYL}$ collected and analyzed data, $\mathrm{XYL}$ and WZ wrote the paper; $\mathrm{WZ}$ and WWZ revised the paper. All authors read and approved the final manuscript.

\section{Acknowledgments}

None.

\section{References}

1. Iannuccilli JD, Cronan JJ, Monchik JM. Risk for malignancy of thyroid nodules as assessed by sonographic criteria: the need for biopsy. J Ultrasound Med. 2004; 23:1455-64. 
2. Wiest PW, Hartshorne MF, Inskip PD, Crooks LA, Vela BS, Telepak RJ, et al. Thyroid palpation versus high-resolution thyroid ultrasonography in the detection of nodules. J Ultrasound Med. 1998; 17:48796.

3. Davies L, Welch HG. Increasing incidence of thyroid cancer in the United States, 1973-2002. JAMA. 2006; 295:2164-7.

4. Roman S, Lin R, Sosa J A. Prognosis of medullary thyroid carcinoma: demographic, clinical, and pathologic predictors of survival in 1252 cases. Cancer. 2006; 107:2134-42.

5. Girelli ME, Nacamulli D, Pelizzo MR, De Vido D, Mian C, Piccolo M, et al. Medullary thyroid carcinoma: clinical features and long-term follow-up of seventy-eight patients treated between 1969 and 1986. Thyroid. 1998; 8:517-23.

6. Modigliani E, Cohen R, Campos JM, Conte-Devolx B, Maes B, Boneu A, et al. Prognostic factors for survival and for biochemical cure in medullary thyroid carcinoma: results in 899 patients. The GETC Study Group. Groupe d'etude des tumeurs a calcitonine. Clin Endocrinol (Oxf). 1998; 48:265-73.

7. Zhou L, Chen B, Zhao M, Zhang H, Liang B. Sonographic Features of Medullary Thyroid Carcinomas According to Tumor Size. J Ultrasound Med. 2015; 34:1003-9.

8. Xia Y, Wang L, Jiang Y, Dai Q, Li X, Li W. Sonographic appearance of primary thyroid lymphomapreliminary experience. PLoS One. 2014; 9:e114080.

9. Choi N, Moon WJ, Lee JH, Baek JH, Kim DW, Park SW. Ultrasonographic findings of medullary thyroid cancer: differences according to tumor size and correlation with fine needle aspiration results. Acta Radiol. 2011; 52:312-6.

10. Lee S, Shin JH, Han BK, Ko EY. Medullary thyroid carcinoma: comparison with papillary thyroid carcinoma and application of current sonographic criteria. AJR Am J Roentgenol. 2010; 194:1090-4.

11. Kim SK, Park I, Woo JW, Lee JH, Choe JH, Kim JH, et al. Predictive factors for lymph node metastasis in papillary thyroid microcarcinoma. Ann Surg Oncol. 2016; 23:2866-73.

12. Ng SC, Kuo SF, Chen ST, Hsueh C, Huang BY, Lin JD. Therapeutic Outcomes of Patients with Multifocal Papillary Thyroid Microcarcinomas and Larger Tumors. Int J Endocrinol. 2017; 2017:4208178.

13. So YK, Kim MW, Son YI. Multifocality and bilaterality of papillary thyroid microcarcinoma. Clin Exp Otorhinolaryngol. 2015; 8:174-8.

14. Kim JY, Jung EJ, Park T, Jeong SH, Jeong CY, Ju YT, et al. Impact of tumor size on subclinical central lymph node metastasis in papillary thyroid microcarcinoma depends on age. World J Surg Oncol. 2015; $13: 88$.

15. Wang WH, Xu SY, Zhan WW. Clinicopathologic Factors and Thyroid Nodule Sonographic Features for Predicting Central Lymph Node Metastasis in Papillary Thyroid Microcarcinoma: A Retrospective Study of 1204 Patients. J Ultrasound Med. 2016; 35:2475-2481.

16. Yin X, Liu C, Guo Y, Li X, Shen N, Zhao X, et al. Influence of tumor extent on central lymph node metastasis in solitary papillary thyroid microcarcinomas: a retrospective study of 1092 patients. World J Surg Oncol. 2017; 15:133. 
17. Yoon SJ, Yoon DY, Chang SK, Seo YL, Yun EJ, Choi CS, et al. "Taller-than-wide sign" of thyroid malignancy: comparison between ultrasound and CT. Ajr Am J Roentgenol. 2010; 194:W420-4.

18. Zhang XL, Qian LX. Ultrasonic features of papillary thyroid microcarcinoma and nonmicrocarcinoma. Exp Ther Med. 2014; 8:1335-1339.

19. Ha SM, Baek JH, Kim JK. Detection of Malignancy Among Suspicious Thyroid Nodules $<1 \mathrm{~cm}$ on Ultrasound with Various Thyroid Image Reporting and Data Systems. Thyroid. 2017; 27:1307-1315.

20. Liu M, Liu Z, Hou Y, Men YM, Zhang YX, Gao LY, et al. Ultrasonographic characteristics of medullary thyroid carcinoma: a comparison with papillary thyroid carcinoma. 2017; 8:27520-27528.

21. Cai S, Liu H, Li WB, Ouyang YS, Zhang B, Li P, et al. Ultrasonographic features of medullary thyroid carcinoma and their diagnostic values. Chin Med J (Engl). 2010; 123:3074-8.

22. Kim SH, Kim BS, Jung SL, Lee JW, Yang PS, Kang BJ, et al. Ultrasonographic findings of medullary thyroid carcinoma: a comparison with papillary thyroid carcinoma. Korean J Radiol. 2009; 10:101-5.

23. Trimboli P, Nasrollah N, Amendola S, Rossi F, Ramacciato G, Romanelli F, et al. Should we use ultrasound features associated with papillary thyroid cancer in diagnosing medullary thyroid cancer?. Endocr J. 2012; 59:503-8.

24. Passe TJ, Bluemke DA, Siegelman SS. Tumor angiogenesis: tutorial on implications for imaging. Radiology. 1997; 203:593-600.

25. Lai X, Liu M, Xia Y, Wang L, Bi Y, Li X, et al. Hypervascularity is more frequent in medullary thyroid carcinoma:Compared with papillary thyroid carcinoma. Medicine (Baltimore). 2016; 95: e5502.

\section{Tables}

Table 1. The clinical characteristics of MTMCs and PTMCs groups

\begin{tabular}{llll}
\hline Parameter & MTMC & PTMC & P \\
\hline $\begin{array}{l}\text { No. of nodules } \\
\text { No. of patients }\end{array}$ & 46 & 136 & \\
$\begin{array}{c}\text { Age(y) } \\
\text { mean }\end{array}$ & 41 & 104 & \\
$\quad$ range & $41.78 \pm 16.39$ & $44.07 \pm 10.43$ & 0.323 \\
$\begin{array}{c}\text { Gender } \\
\text { male }\end{array}$ & $14-80$ & $24-71$ & \\
$\quad$ female & $18(43.90 \%)$ & $34(32.69 \%)$ & 0.205 \\
$\begin{array}{c}\text { Size(mm) } \\
\text { mean }\end{array}$ & $23(56.10 \%)$ & $70(67.31 \%)$ & \\
$\quad$ range & & & \\
$\begin{array}{c}\text { Multiplicity } \\
\text { single }\end{array}$ & $6.30 \pm 2.27$ & $6.06 \pm 2.16$ & 0.605 \\
$\quad$ multifocal & $2.0-9.91$ & $1.5-10$ & \\
Lymph node metastases & $5(12.20 \%)$ & $31(29.81 \%)$ & \\
$\quad$ none & & & 0.027 \\
$\quad$ present & $26(63.41 \%)$ & $83(79.81 \%)$ & \\
\hline
\end{tabular}


Table 2. The US characteristics of MTMCs and PTMCs on Univariate Analysis

\begin{tabular}{|c|c|c|c|}
\hline Parameter & MTMC $(\mathrm{N}=46)$ & PTMC $(\mathrm{N}=136)$ & $\mathrm{P}$ \\
\hline Location & & & 0.86 \\
\hline upper pole & $24 / 52.17 \%$ & $73 / 53.68 \%$ & \\
\hline lower pole & $22 / 47.83 \%$ & $63 / 46.32 \%$ & \\
\hline Shape & & & 0 \\
\hline ovoid to round & $25 / 54.35 \%$ & $13 / 9.56 \%$ & 0 \\
\hline taller than wide & 9/19.57\% & $81 / 59.56 \%$ & 0 \\
\hline irregular & $12 / 26.09 \%$ & $42 / 30.88 \%$ & 0.538 \\
\hline Boundary & & & 0 \\
\hline clear & $26 / 56.52 \%$ & $19 / 13.97 \%$ & \\
\hline unclear & $20 / 43.48 \%$ & $117 / 86.03 \%$ & \\
\hline Margin & & & 0.101 \\
\hline smooth & $17 / 36.96 \%$ & $37 / 27.21 \%$ & 0.211 \\
\hline spiculated & $10 / 21.74 \%$ & $53 / 38.97 \%$ & 0.034 \\
\hline poorly defined & $19 / 41.30 \%$ & $46 / 33.82 \%$ & 0.36 \\
\hline Peripheral halo ring & & & 0.595 \\
\hline absent & $41 / 95.65 \%$ & $133 / 97.79 \%$ & \\
\hline present & $2 / 4.35 \%$ & $3 / 2.21 \%$ & \\
\hline Echogenicity & & & 0.727 \\
\hline marked hypoechoic & $10 / 21.74 \%$ & $33 / 24.26 \%$ & \\
\hline hypoechoic & $36 / 78.26 \%$ & $103 / 75.74 \%$ & \\
\hline hyperechoic or isoechoic & 0 & 0 & \\
\hline Composition & & & 0.004 \\
\hline solid & $40 / 86.96 \%$ & $134 / 98.53 \%$ & \\
\hline$>50 \%$ solid & $6 / 13.04 \%$ & $2 / 1.47 \%$ & \\
\hline Calcification & & & 0.002 \\
\hline absent & $31 / 67.39 \%$ & $87 / 63.97 \%$ & 0.674 \\
\hline microcalcification & $5 / 10.87 \%$ & $40 / 29.41 \%$ & 0.012 \\
\hline macrocalcification & $10 / 21.74 \%$ & $9 / 6.62 \%$ & 0.004 \\
\hline Vascularity & & & 0.000 \\
\hline avascularity & $19 / 41.30 \%$ & $109 / 80.15 \%$ & \\
\hline hypervascularity & $27 / 58.70 \%$ & $27 / 19.85 \%$ & \\
\hline
\end{tabular}

Table 3. The US characteristics of MTMCs and PTMCs on Multivariate Logistic Regression Analysis 


\begin{tabular}{llllll}
\hline \multicolumn{1}{c}{ Variable } & \multicolumn{5}{c}{ Multivariate Analysis } \\
\cline { 2 - 6 } & \multicolumn{1}{c}{ Bals $\chi^{2}$} & Odds Ratio & 95\% CI & P \\
\hline Shape & \multicolumn{5}{c}{16.876} \\
$\quad$ Ovoid to round vs Irregular & 1.391 & 5.792 & 4.018 & $1.295-12.473$ & 0.016 \\
$\quad$ Taller than wide vs Irregular & 2.602 & 16.869 & 13.488 & $3.897-46.681$ & 0 \\
Boundry & -0.623 & 1.735 & 0.536 & $0.212-1.356$ & 0.188 \\
Composition & -2.043 & 4.579 & 0.13 & $0.020-0.842$ & 0.032 \\
Calcification & & 8.808 & & & 0.012 \\
$\quad$ Microcalcification vs absent & -0.829 & 1.543 & 0.436 & $0.118-1.615$ & 0.214 \\
$\quad$ Macrocalcification vs absent & -2.465 & 8.314 & 0.085 & $0.016-0.454$ & 0.004 \\
Vascularity & 2.377 & 21.434 & 10.778 & $3.939-29.488$ & 0 \\
\hline
\end{tabular}

\section{Figures}

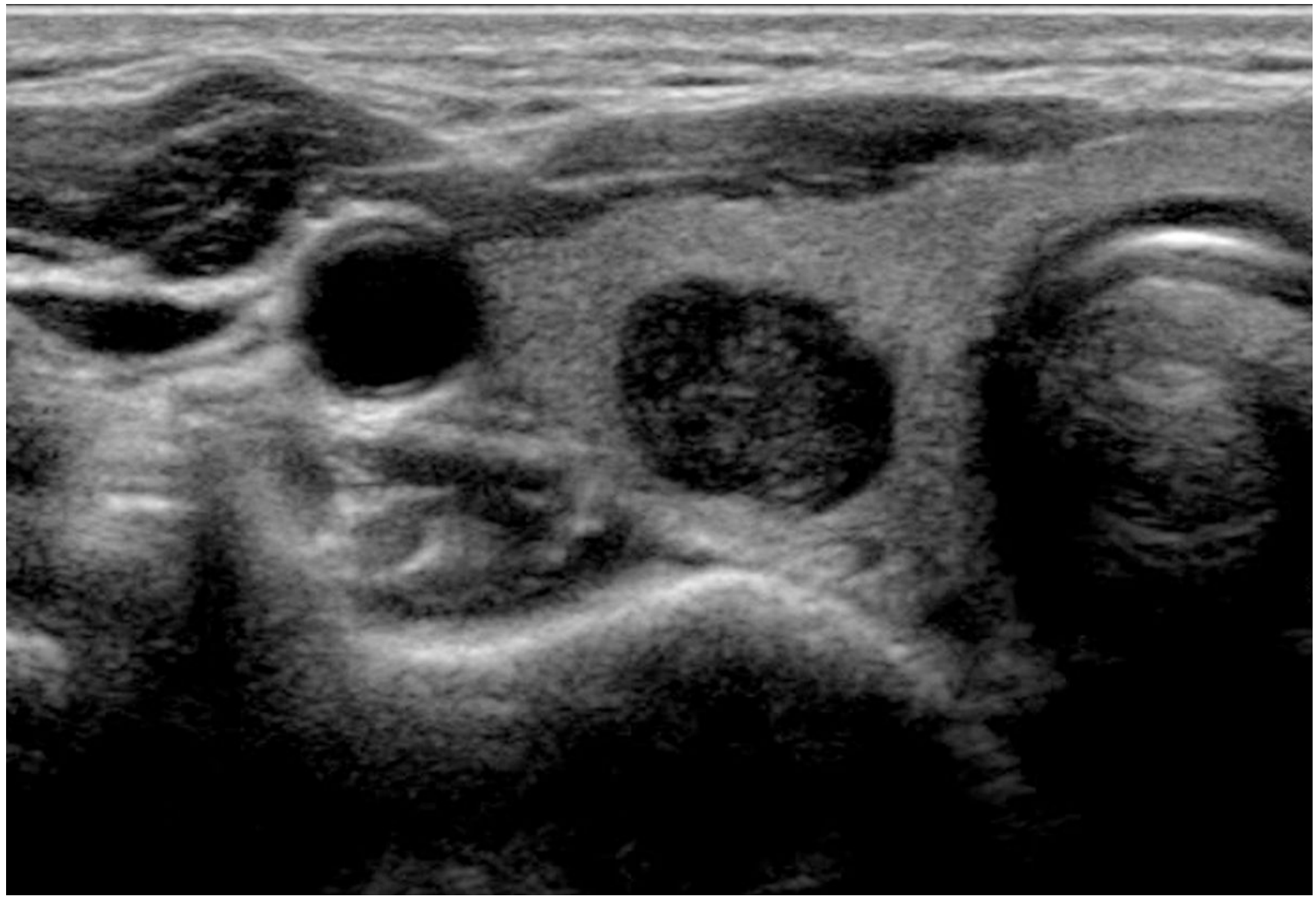

Figure 1

The sonographic features of ovoid to round shape, marked hypoechogenicity, solid composition, and smooth margin were shown on a transverse ultrasound image from a 40-year-old female with MTMC. 


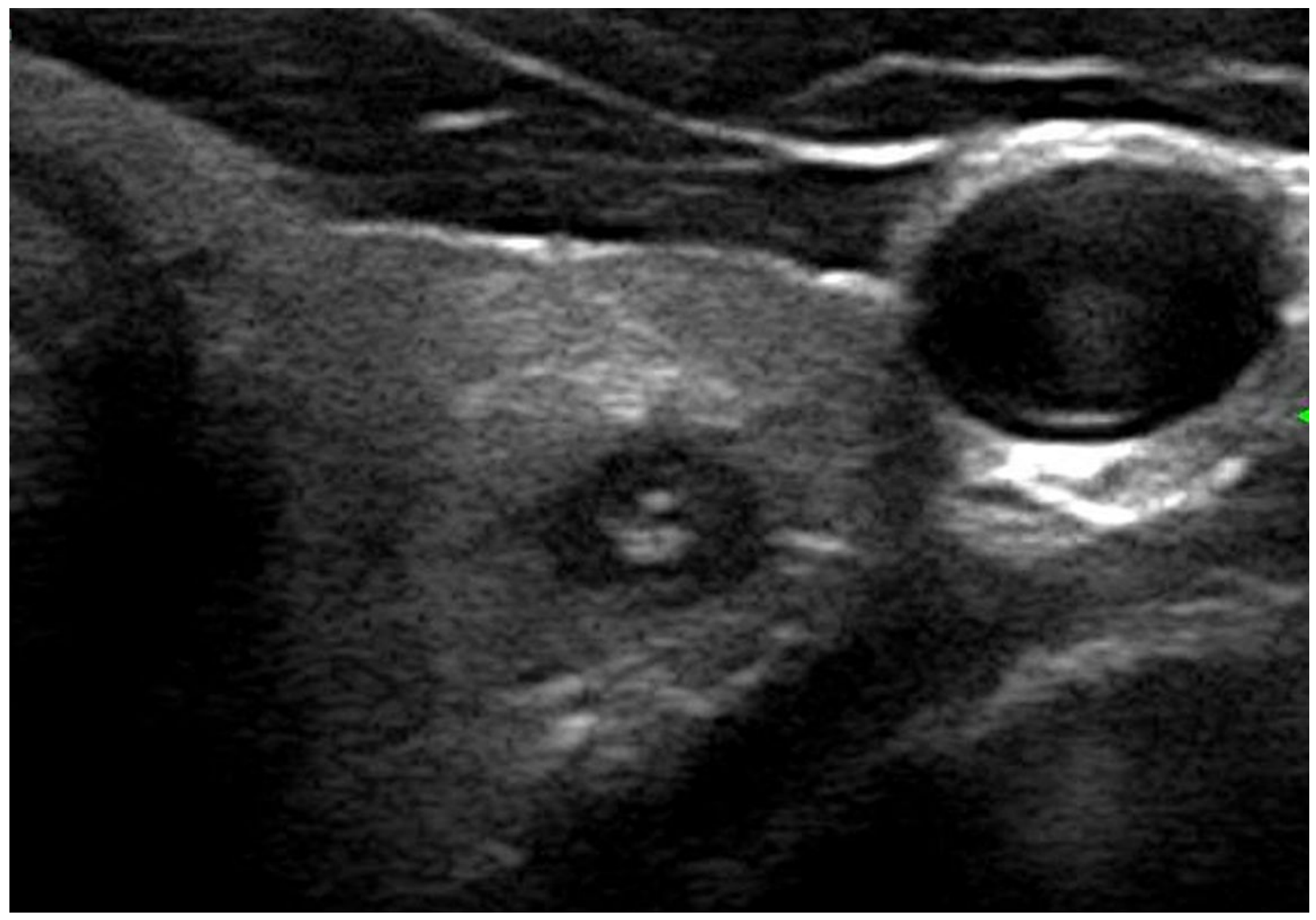

Figure 2

The transverse sonographic image of MTMC in a 68-year-old male showed hypoechogenicity, ovoid to round shape, and macrocalcifications. 


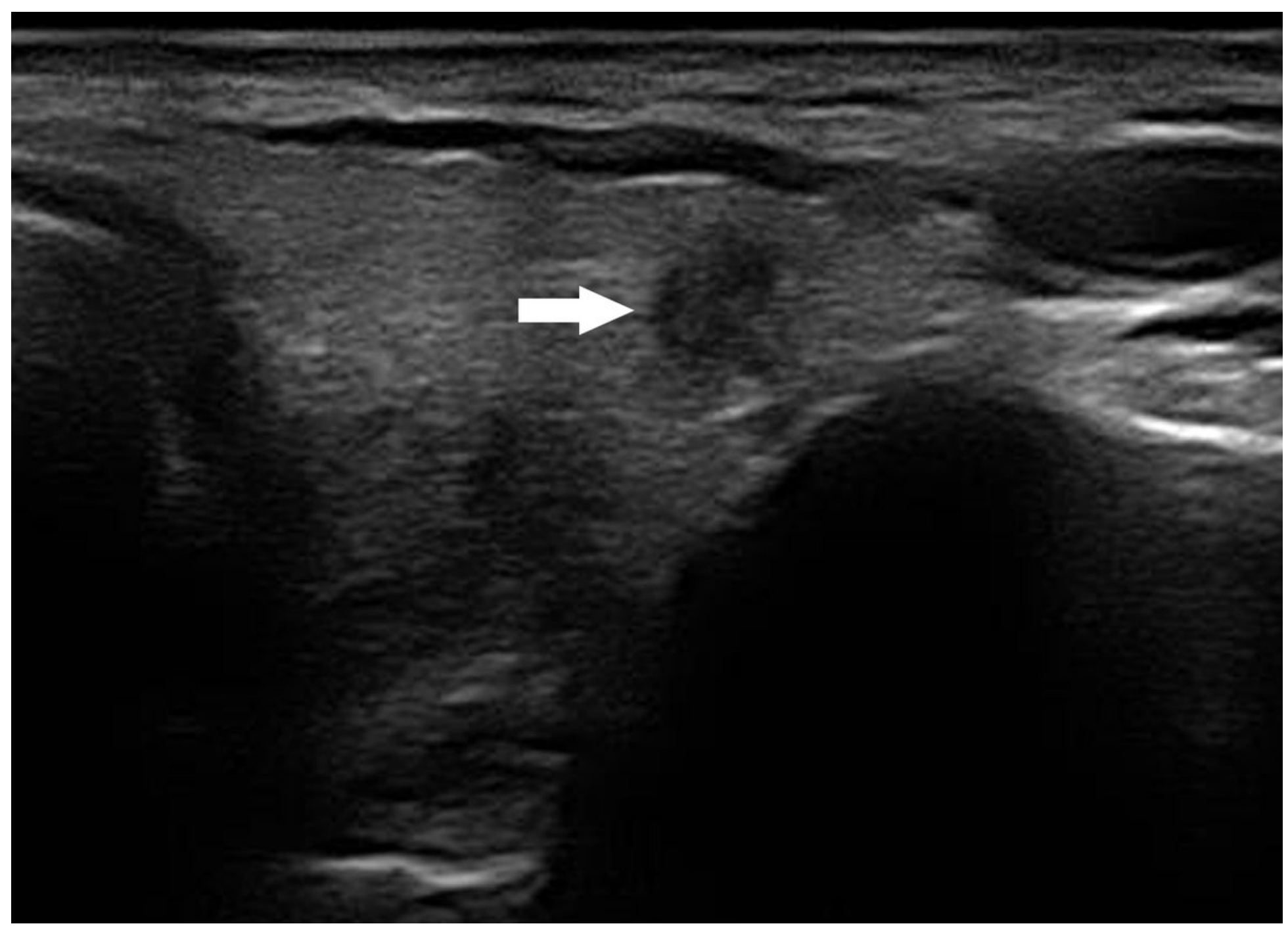

\section{Figure 3}

The MTMC nodule transverse sonographic image of a 76-year-old female displayed taller than wide shape, poorly defined margin, and hypoechogenicity. 


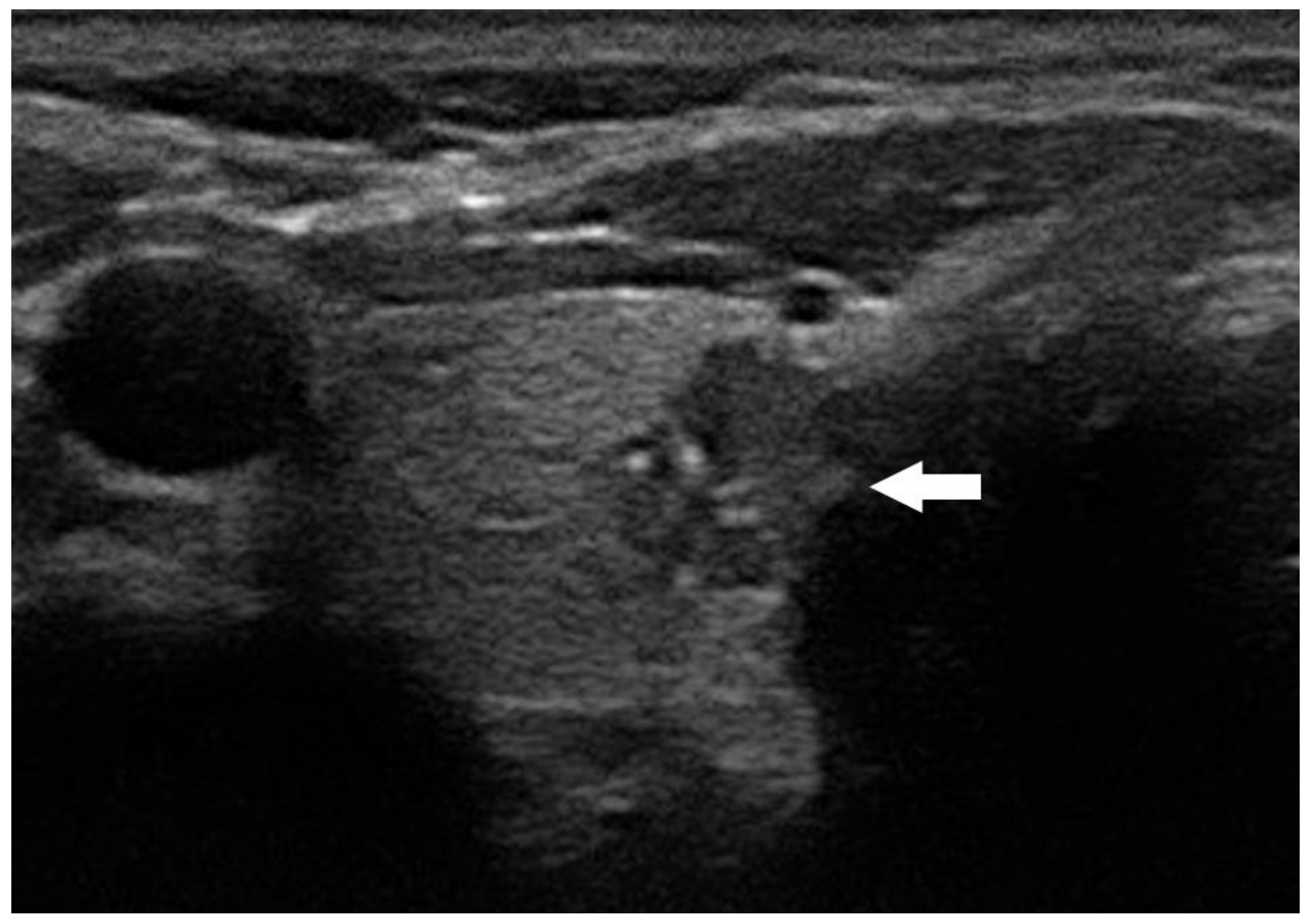

Figure 4

The PTMC transverse ultrasound image of a 28-year-old female showed taller than wide shape, poorly defined margin, and microcalcifications. 


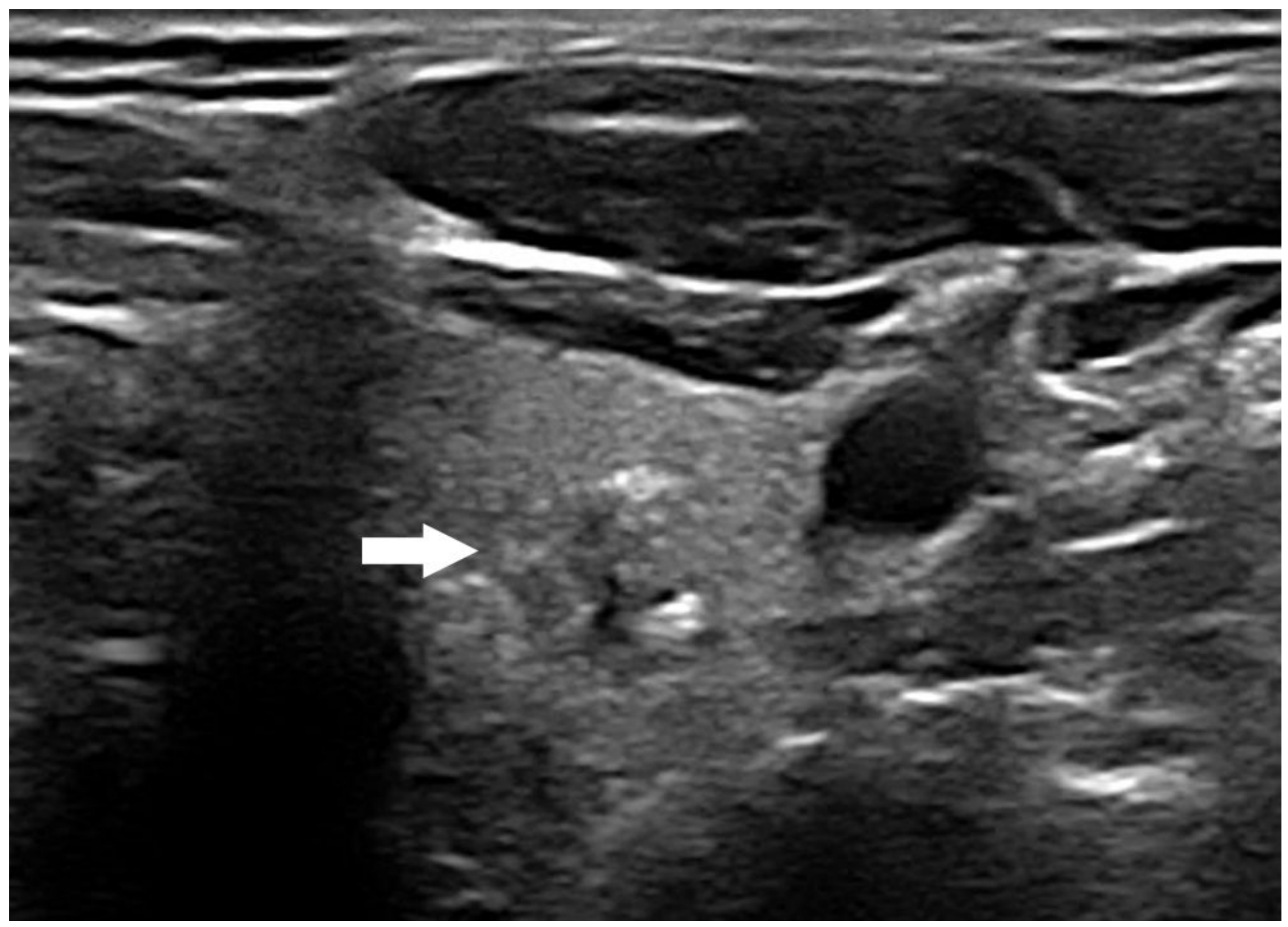

\section{Figure 5}

PTMC in a 36-year-old male. The transverse ultrasound image showed ovoid to round shape, unclear boundary and macrocalcifications. 


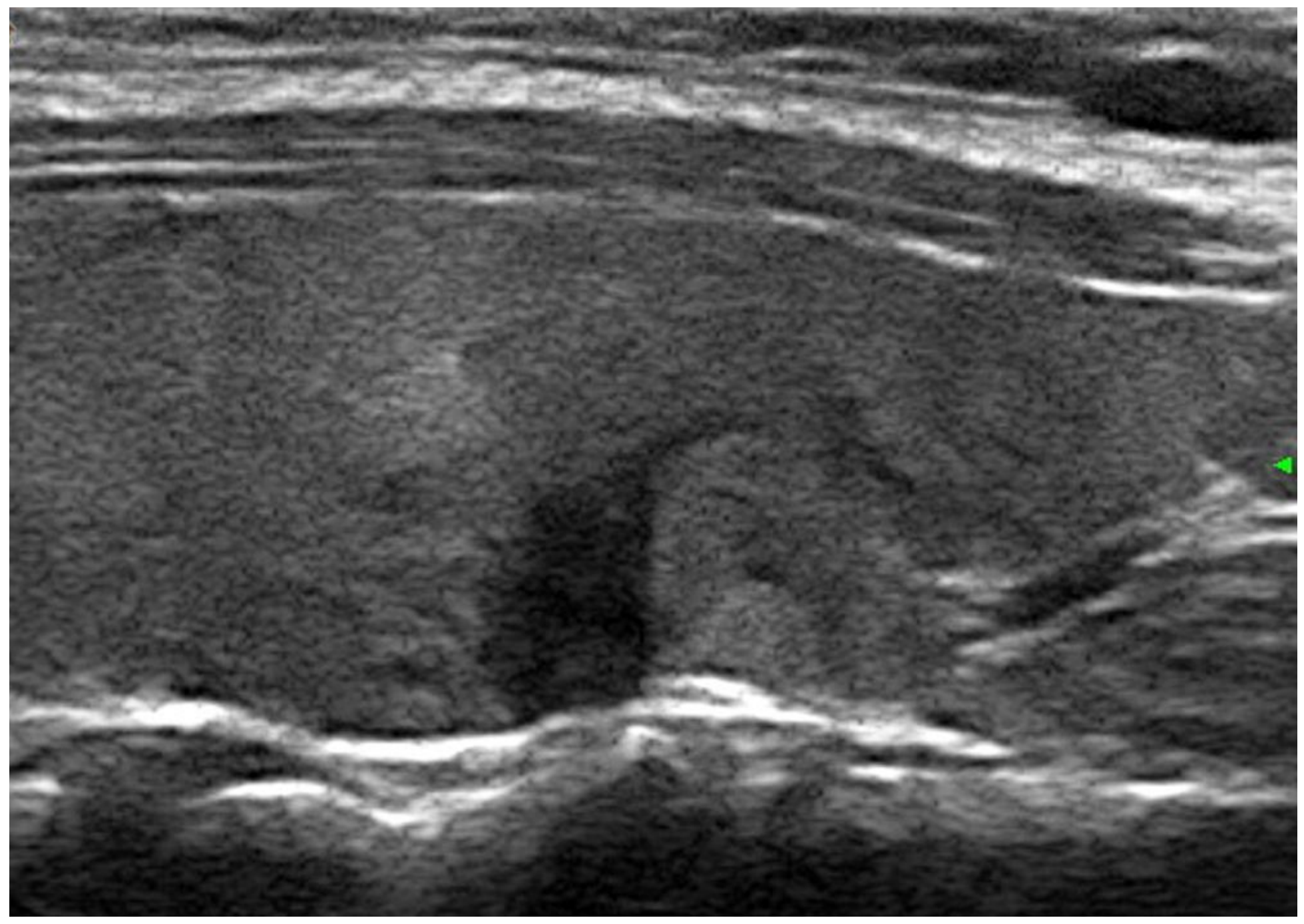

Figure 6

A 40-year-old female with PTMC. The longitudinal sonogram of the tumor presented a taller than wide shape and speculated margin. 